\title{
Microwave-induced water flow in a microchannel built on a coplanar waveguide
}

Cite as: J. Appl. Phys. 110, 064912 (2011); https://doi.org/10.1063/1.3641516

Submitted: 31 March 2011 . Accepted: 13 August 2011 . Published Online: 26 September 2011

A. Khayari, M. Medrano, E. Verlage, M. C. Velázquez-Ahumada, M. J. Freire, and A. Ramos

A

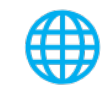

\section{ARTICLES YOU MAY BE INTERESTED IN}

Microwave-induced water flows in microsystems

Applied Physics Letters 94, 024104 (2009); https://doi.org/10.1063/1.3070521

Nickel: A very fast diffuser in silicon

Journal of Applied Physics 113, 204906 (2013); https://doi.org/10.1063/1.4807799

Microwave frequency sensor for detection of biological cells in microfluidic channels

Biomicrofluidics 3, 034103 (2009); https://doi.org/10.1063/1.3187149

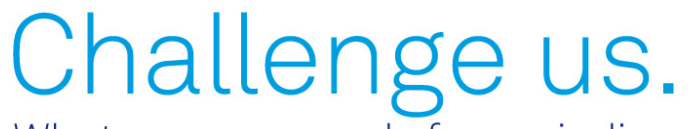

What are your needs for periodic signal detection?

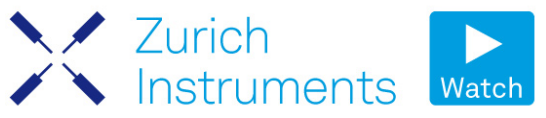

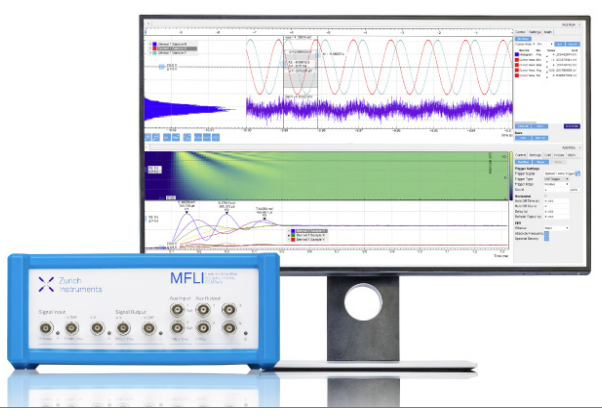

110, 064912 


\title{
Microwave-induced water flow in a microchannel built on a coplanar waveguide
}

\author{
A. Khayari, M. Medrano, E. Verlage, M. C. Velázquez-Ahumada, M. J. Freire, \\ and A. Ramos \\ Dpto. Electronica y Electromagnetismo, Facultad de Fisica, Universidad de Sevilla, Sevilla 41012 Spain
}

(Received 31 March 2011; accepted 13 August 2011; published online 26 September 2011)

\begin{abstract}
We present experimental and numerical investigations of water flow in a microsystem induced by microwave electric fields. Microwave dielectric heating induces gradients of temperature which produce spatial variations in mass density and dielectric permittivity that lead to buoyancy and dielectric forces in the liquid, respectively. The experimental system consists of a microchannel, filled with water, which is built on top of a coplanar waveguide operating in the fundamental transversal electromagnetic (TEM) mode at frequencies in the range $1-4 \mathrm{GHz}$. The flow originated by standing waves is studied. Maxima and minima of electric field amplitude lead to maxima and minima of fluid flow. This observation allows us to measure the TEM wavelength and good agreement is found with the theoretical results for the TEM mode inside the microchannel. We also present three dimensional finite-element calculations of the electric, temperature and fluid velocity fields in the microchannel. In a first approach, the calculations are performed using the equations in the limit of small temperature variations, which allows us to decouple the electrical, mechanical and thermal equations. These calculations show a good agreement with the velocity profiles. Subsequently, the effect of considering finite increments of temperature is taken into account and the new numerical results improve the quantitative comparison with experimental velocities. (C) 2011 American Institute of Physics.
\end{abstract} [doi:10.1063/1.3641516]

\section{INTRODUCTION}

Microwave (MW) heating of liquids have been utilized in a wide range of industrial processes. It offers numerous advantages such as short start-up time, rapid heating, energy efficiency, and precise process control. ${ }^{1}$ Microwave electric fields have recently been used in the lab-on-a-chip technology for detection of biomolecules ${ }^{2}$ or cells, ${ }^{3}$ as well as for controlled dielectric heating of water inside a microchannel ${ }^{4}$ or of drops ${ }^{5}$ in microdevices. Recently, electric fields at microwave frequencies have been demonstrated experimentally to generate water flows in microfluidic devices. ${ }^{6}$ This fluid motion may be a limiting factor for the applications of microwaves in microsystems. On the other hand, the lab-ona-chip technology may take advantage of the water flows generated by microwave electric fields. The microwaveinduced microflows have been analyzed numerically ${ }^{7}$ and the computed flow was found to be in semi-quantitative agreement with the experimental observations. ${ }^{6}$

The theoretical model employed to analyze these flows assumes that gradients in permittivity and mass density generated due to microwave dielectric heating of water are responsible of the observed fluid flow in microsystems. The liquid motion appears as a consequence of both buoyancy $\left(\mathbf{f}_{\mathbf{g}}=\Delta \rho_{m} \mathbf{g}\right)$ and dielectric forces $\left(\mathbf{f}_{\mathbf{E}}=-\frac{1}{2} \mathbf{E}^{2} \nabla \varepsilon\right)$, where $\mathbf{g}$ is the acceleration due to gravity, $\mathbf{E}$ is the electric field, and $\rho_{m}$ and $\varepsilon$ are, respectively, the mass density and the dielectric permittivity of the liquid. For microfluidic applications, microwave water actuation presents the advantage of being quite independent of electrical conductivity, as happens for signal frequencies under $3 \mathrm{GHz}$ and conductivities below 3 $\mathrm{S} / \mathrm{m}$. Therefore, most biological fluids could be actuated with the same behavior using the same applied signal.

In this paper, we intend to perform a more quantitative comparison between experiments and theoretical modeling of the water flow in a microdevice generated by microwave fields since, in Refs. 6 and 7, the comparison was mainly qualitative. For this reason, we have improved the experimental setup so to know the microwave power consumed in the water sample and the numerical simulations were performed in 3D including the TEM mode. In addition, we have established standing waves in the device, so that the boundary conditions for the electric field are well defined.

The paper is organized as follows. First, we describe the experimental setup and show the experimental results of water flow induced in the microchannel by standing waves of frequencies in the range 1-4 GHz. Then we present the theoretical background that leads to the numerical model used for the simulations. We calculate three dimensional (3D) distributions of the electric field $\mathbf{E}$, temperature $T$ and flow velocity $\mathbf{v}$. In a first approach, we consider that the increments of temperature are small so that the variations of permittivity and mass density with $T$ are only important in the body force expressions (Boussinesq approximation). ${ }^{8}$ This allows us to decouple the electrical, thermal, and mechanical equations. The numerical results using this approximation are then compared with our experimental data. Finally, we perform new calculations in order to analyze the effect of including the variations of physical properties, such as electrical permittivity and viscosity, with $T$ in all the equations. 


\section{EXPERIMENTAL OBSERVATIONS}

\section{A. Experimental setup}

The system of our study is a coplanar waveguide with a microchannel filled with water on top of it. The coplanar waveguide consists of three coplanar electrodes (see Fig. 1), which are $20 \mathrm{~mm}$ long with parallel edges and photoetched on a ROGERS PCB (Printed Circuit Board). The substrate layer of the PCB is $635 \mu \mathrm{m}$ thick, and the copper layer used to design the electrodes is $30 \mu \mathrm{m}$ thick. The inner electrode is $125 \mu \mathrm{m}$ wide, the two outer electrodes are $5.5 \mathrm{~mm}$ wide, and outer electrodes are symmetrically separated by $175 \mu \mathrm{m}$ gaps from the inner one. A squared chamber is built with PDMS (polydimethylsiloxane, soft polymer used in microfluidics) upon the PCB board and covered by a thin glass layer, $1.2 \mathrm{~mm}$ thick, at a height of $270 \mu \mathrm{m}$ from the electrodes.

Two coplanar waveguides were built with two different terminations: open ended and short ended (see Fig. 2). Both configurations support a standing wave pattern when excited with the fundamental TEM mode, and will provide equidistant maxima and minima of field along the longitudinal direction (z-direction in Figs. 1 and 2). The position of maxima and minima can be predicted by using transmission line theory and the predictions can be correlated with the experimental observation of fluid velocity along distance in the waveguide. Therefore, these configurations are used because they establish standing waves, with definite boundary conditions: for the short-ended configuration the electric field is zero at the end; for the open-ended configuration the electric field is maximum at the end. $\mathrm{KCl}$ water solutions with fluorescent particle tracers $(500 \mathrm{~nm}$ diameter) are pipetted into the chamber, and a microscope is used to observe the fluid flow from the top.

Microwave actuation is performed by means of an incident sinusoidal wave in fundamental TEM mode and the microwave energy is transmitted along the $z$-direction (the channel length direction). MW signals are generated with a N9192 A Fieldfox network analyzer from Agilent Tech., and the signal power is amplified and controlled by an amplifier (AR 5S164) with a working frequency range of 0.8 to 4.2 $\mathrm{GHz}$ and power range from 0 to $5 \mathrm{~W}$. During the application of the MW signals, fluid flow is observed in the microchan-

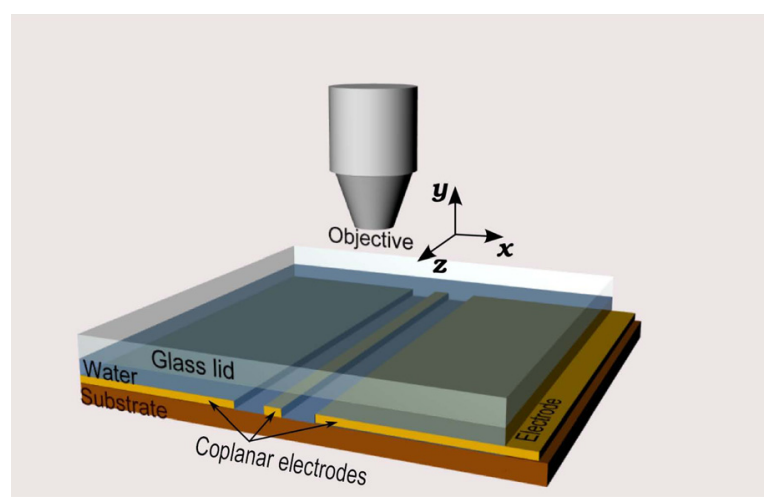

FIG. 1. (Color online) Sketch of the system under study (not at scale). The signal electrode is in the middle of the two grounded electrodes.

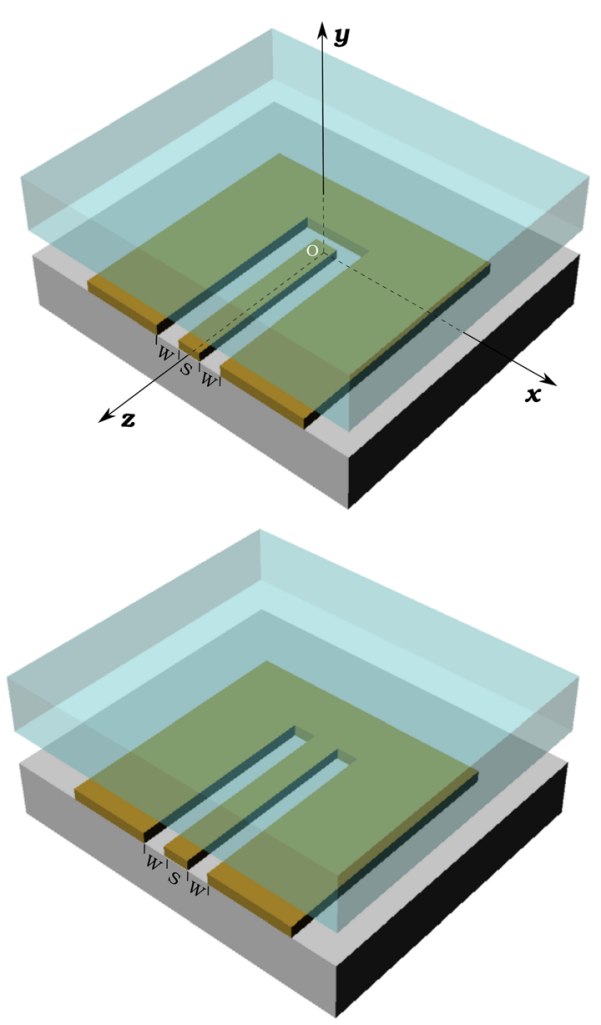

FIG. 2. (Color online) Open-end and short-end configurations of a coplanar waveguide. Parameters $S$ and $W$ are, respectively, the width of the source electrode and the interelectrodes gap. Refer to Fig. 3 for the complete geometry parameters.

nel. The conductivity of the $\mathrm{KCl}$ water solution was fixed to $0.04 \mathrm{~S} / \mathrm{m}$, other properties of water and materials employed are summarized in Table I.

The reflection coefficient $\left(S_{11}\right)$ of the water-loaded coplanar waveguide was measured with the same N9192 A network analyzer in a frequency range from 1 to $4 \mathrm{GHz}$. The incident power $P_{0}$ provided by the microwave amplifier was measured in the same range of frequencies with an Agilent U2000 Series USB power sensor. Thus, the real power delivered to the coplanar waveguide at each frequency was obtained as the difference between the incident power and the reflected power, that is, $P_{0}\left(1-\left|S_{11}\right|^{2}\right)$. Since there is a reflecting boundary condition at the end of the microchannel (open or short), we assume that all the power delivered to the coplanar waveguide was consumed by losses in the microchannel.

Liquid flow is recorded in video format with a CCD camera mounted on a microscope. Post-processing of the recorded videos is performed with ImageJ, a free-ware program. ${ }^{9}$

TABLE I. Material properties (at $\left.20^{\circ} \mathrm{C}\right) . \varepsilon_{r}^{\prime}$ : relative permittivity, $k$ : thermal conductivity and $c_{p}$ : specific heat at constant pressure.

\begin{tabular}{lccc}
\hline \hline Material & $\varepsilon_{r}^{\prime}$ & $k \mathrm{~W} /(\mathrm{m} \cdot \mathrm{K})$ & $c_{p} \mathrm{~J} /(\mathrm{kg} \cdot \mathrm{K})$ \\
\hline Water & 77.88 & 0.6 & 4181 \\
Glass & 4.6 & 0.14 & 840 \\
Substrate & 10 & 0.66 & 930 \\
Copper & - & 386 & 385 \\
Air & 1 & 0.025 & 1012 \\
\hline \hline
\end{tabular}




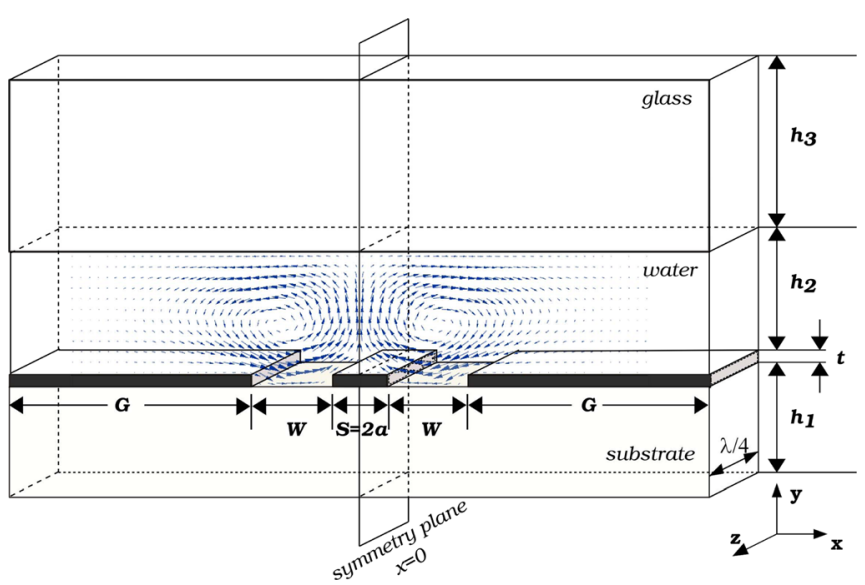

FIG. 3. (Color online) Computation domain. Parameters shown are as follows (in $\mu \mathrm{m}$ ): copper electrodes: $S=2 a=125, t=30, G=5500$; interelectrodes gap: $W=175$; substrate height: $h_{1}=635$, water: $h_{2}=270$, glass: $h_{3}=1200$. Two layers of air, $1200 \mu \mathrm{m}$ height each, are added to the computation domain: under the substrate and above the glass to account for heat exchange with surrounding air.

\section{B. Experimental results}

During the application of the microwave signal, fluid flow is observed. The velocity vector lies mainly in planes of constant $z$, i.e., $\mathbf{v}(x, y, z) \approx v_{x} \mathbf{e}_{x}+v_{y} \mathbf{e}_{y}$. The flow in each $z$-plane is composed of two symmetric rolls with liquid going up in the center electrode at symmetry plane $x=0$ (see Fig. 3). The intensity of the fluid motion varies with $z$, and maxima and minima of motion are clearly observed, which correspond to maxima and minima of electric field.

The flow velocity is measured by tracking the trajectory of the fluorescent particles. The position of around ten particles is determined in different video-frames and the velocity measurement is taken as the average particle velocity and the uncertainty is given by the dispersion amplitude.

Figure 4 depicts measured velocities of the fluid in short-end configuration at the cross section $z=4 \mathrm{~mm}$. The plot shows velocity as a function of the channel width $x-a$ ( $a=62.5 \mu \mathrm{m}$ is the half width of the source electrode) at two different heights: at the electrode level (coordinate $y=30$ $\mu \mathrm{m})$ and at $50 \mu \mathrm{m}$ above electrode (coordinate $y=80 \mu \mathrm{m}$ ). Together with experimental data, numerical results are shown for comparison purpose, although the theoretical model and numerical simulations will be introduced later in Secs. III, IV, and V. The plots show clearly how the direction and profile of velocity in the cross section is correctly predicted by the model. Nevertheless, at the electrodes level the experimental data show some discrepancy at the right part of the plot, which can be ascribed to the experimental error in locating the electrode edge. A small deviation from this edge gives, numerically, a non-zero velocity.

Figure 5 shows path lines of fluorescent latex particles in a plane at $50 \mu \mathrm{m}$ above electrodes, in the short-end configuration. The fluid flow is visualized at different axial coordinates, and the path length is an indication of the fluid velocity amplitude. As can be seen, at $z=0$ and $8 \mathrm{~mm}$ there is almost no motion, and at $z=4$ and $12 \mathrm{~mm}$ the flow is strong (see Fig. 6 upper plot). In Fig. 6, we plot velocity $-v_{x}$ as a function of coordinate $z$ along the channel length. The
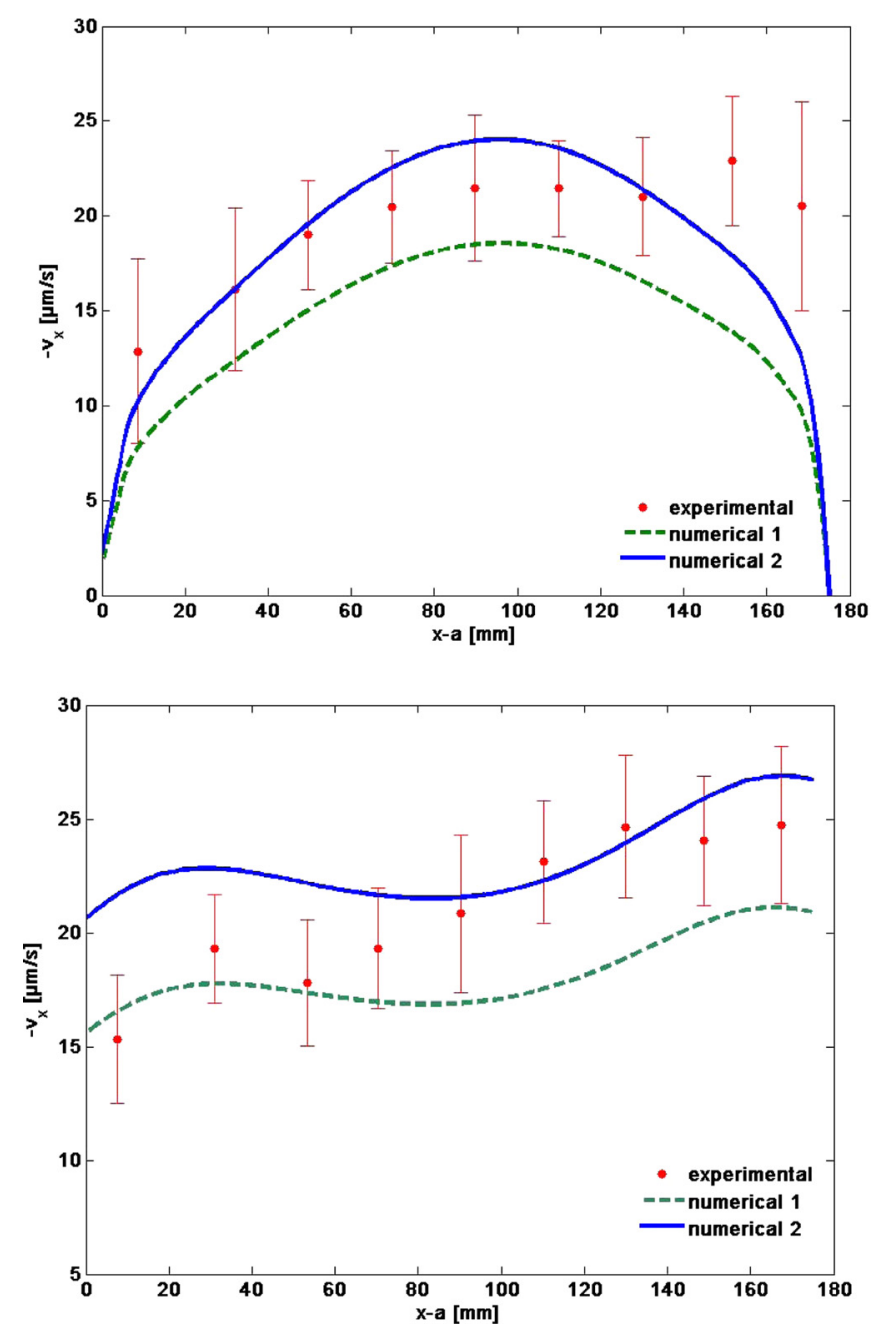

FIG. 4. (Color online) Experimental results for fluid velocity as function of the interelectrodes gap, in the plane $z=4 \mathrm{~mm}$ for frequency $f=3.055 \mathrm{GHz}$, power $P_{0}=358.51 \mathrm{~mW}, a=S / 2=62.5 \mu \mathrm{m}$. Short-end configuration is shown here; the top plot shows velocities at electrode level $(y=30 \mu \mathrm{m})$ and the bottom one, at $50 \mu \mathrm{m}$ above the electrode $(y=80 \mu \mathrm{m})$. Numerical 1 refers to model with $\varepsilon$ and $\eta$ values at $20^{\circ} \mathrm{C}$, while numerical 2 refers to model with $\varepsilon$ and $\eta T$-dependent (see Secs. IV and V).

apparent wavelength in the velocity plots should correspond to half wavelength of the input signal since the velocity amplitude $|\mathbf{v}|$ along the channel coordinate $z$ is an increasing function of the electric field amplitude $|\mathbf{E}|$, i.e., $v(z)$ reaches a maximum for each maximum of the electric field amplitude $E(z)$ (refer to Sec. IV). We recall that the distance between nodes in standing waves is one half of the wavelength. The fit of the experimental data with a sine wave gives an estimation of the experimental value of the wavelength of the input signal: $\lambda_{\exp }=14.0 \mathrm{~mm}$ versus a theoretical $\lambda_{t h}=15.2 \mathrm{~mm}$ for short-end configuration and MW signal of $3.05 \mathrm{GHz}$; and $\lambda_{\exp }=20.5 \mathrm{~mm}$ versus a theoretical $\lambda_{\text {th }}=19.4 \mathrm{~mm}$ for the open-end configuration and MW signal of $2.38 \mathrm{GHz}$. As we can see, the agreement is very good with the theoretical prediction of $\lambda$ using transmission line theory (see Sec. III C). The same agreement was found at different frequencies.

Figure 7 shows measured velocities as a function of input power $P_{0}$ for different values of $z$ in open and short configurations. The frequency is fixed to $3.05 \mathrm{GHz}$ for the short-end 

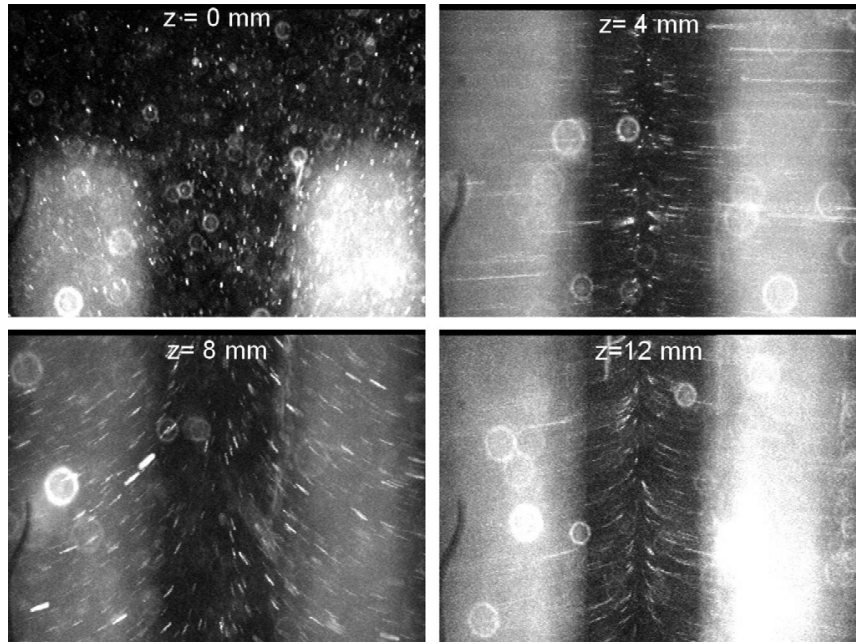

FIG. 5. Flow visualization using fluorescent particle tracers in short-end configuration for different axial coordinate $z(f=3.05 \mathrm{GHz}, V=5.17 \mathrm{~V})$. Maximum velocities are located near $z=4$ and $12 \mathrm{~mm}$ (refer to Fig. 6 upper plot).

configuration and to $2.38 \mathrm{GHz}$ for the open-end configuration. We can see that the velocity amplitude increases for increasing input power. For low values of input power the velocity seems to be proportional to $P_{0}$, but for higher values it approaches $P_{0}^{2}$. This seems to originate from the expected transition from fluid flow generated by buoyancy (proportional to $P_{0}$ ) to fluid flow generated by dielectric forces (proportional to $P_{0}^{2}$ ) as input power increases (see Sec. III).

\section{THEORETICAL BACKGROUND}

Microwave electric fields generate a non-homogeneous temperature field inside our microchannel by dielectric heating of the water sample. Because material properties of water are functions of temperature, both electrothermal forces due to gradients in electrical permittivity $\varepsilon$ and conductivity $\sigma$ as well as buoyancy forces due to gradients in mass density appear in the liquid bulk and lead to water flow. In a first approximation, we can consider that changes in temperature are small so that the relative increments in permittivity $\Delta \varepsilon / \varepsilon$, conductivity $\Delta \sigma / \sigma$, and mass density $\Delta \rho_{m} / \rho_{m}$ are much less than unity. This approximation allows us to consider that material properties are constant in the equations, and the variations are only important in the expression for the body force (Boussinesq-like approximation).

\section{A. Electric force}

The electrical body force in the fluid bulk is given by ${ }^{10}$

$$
\mathbf{f}_{\mathbf{E}}=\rho \mathbf{E}-\frac{1}{2}|\mathbf{E}|^{2} \nabla \varepsilon+\frac{1}{2} \nabla\left[\rho_{m}\left(\frac{\partial \varepsilon}{\partial \rho_{m}}\right)_{T}|\mathbf{E}|^{2}\right],
$$

where $\rho$ and $\rho_{m}$ are the electrical charge density and mass density, respectively, and $\varepsilon$ is the dielectric permittivity for static fields. Expression 1 is obtained from thermodynamic arguments for static fields. At giga-hertz frequencies, water permittivity is a complex function of frequency $\tilde{\varepsilon}=\varepsilon^{\prime}-i \varepsilon^{\prime \prime}$. We will assume that the force expression is still valid for frequencies much smaller than $16 \mathrm{GHz}$ (the water relaxation
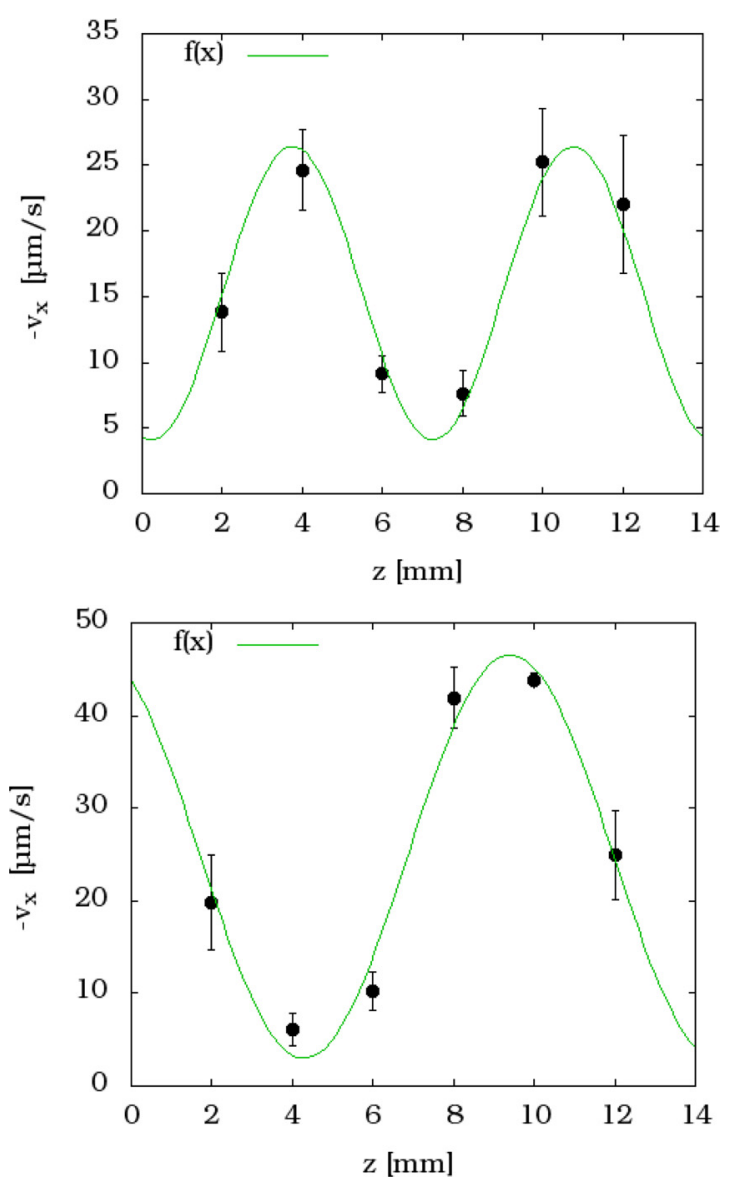

FIG. 6. (Color online) Velocity $v_{x}$ as function of $z$, along the path $(x, y)=(188,80) \mu \mathrm{m}$. Experimental data for short-end on the top at a signal MW frequency of $3.05 \mathrm{GHz}$ and open-end on the bottom at a signal MW frequency of $2.38 \mathrm{GHz}$. A fit of the experimental data with a sine wave is shown in the plots, which gives an estimation of the experimental value of the input signal wavelength: $\lambda=14.0 \mathrm{~mm}$ vs a theoretical $15.2 \mathrm{~mm}$ for short-end configuration and $\lambda=20.5 \mathrm{~mm}$ vs a theoretical $19.4 \mathrm{~mm}$ for the open-end. Note: the apparent wavelength in the plots corresponds to half of the wavelength of the input signal (see Sec. IV).

frequency), using $\varepsilon=\varepsilon^{\prime}$, since the real part of the complex permittivity accounts for the electrical energy stored in the dielectric. Notice that $\varepsilon^{\prime}$ is approximately equal to the static value for frequencies below $5 \mathrm{GHz}$. Under the approximation of small temperature changes, the following force expression can be derived for AC fields: ${ }^{11-13}$

$$
\left\langle\mathbf{f}_{\mathbf{E}}\right\rangle=\frac{1}{2} \Re\left[\frac{\sigma \varepsilon(\alpha-\beta)}{\sigma+i \omega \varepsilon}(\nabla T \cdot \mathbf{E}) \mathbf{E}^{*}-\frac{1}{2} \varepsilon \alpha\left|\mathbf{E}^{2}\right| \nabla T\right],
$$

where $\mathbf{E}^{*}$ is the complex conjugate of the electric field, $\Re[\cdot]$ denotes the real part, $\omega$ is the angular frequency, and $i=\sqrt{-1}$. In the force expression, gradients in $\varepsilon$ and $\sigma$ have been related linearly to $\nabla T$ by $\nabla \varepsilon=\varepsilon \alpha \nabla T$ and $\nabla \sigma=\sigma \beta \nabla T$, where the parameters $\alpha=(1 / \varepsilon)(\partial \varepsilon / \partial T)$ $=-0.004 \mathrm{~K}^{-1}$ and $\beta=(1 / \sigma)(\partial \sigma / \partial T)=-0.02 \mathrm{~K}^{-1} \cdot{ }^{14}$ The ratio between the first and second terms (Coulomb force and dielectric force, respectively) is of the order of

$$
\frac{\text { Coulomb }}{\text { dielectric }} \sim \frac{\sigma \beta}{\alpha \sqrt{(\omega \varepsilon)^{2}+\sigma^{2}}} \ll 1
$$



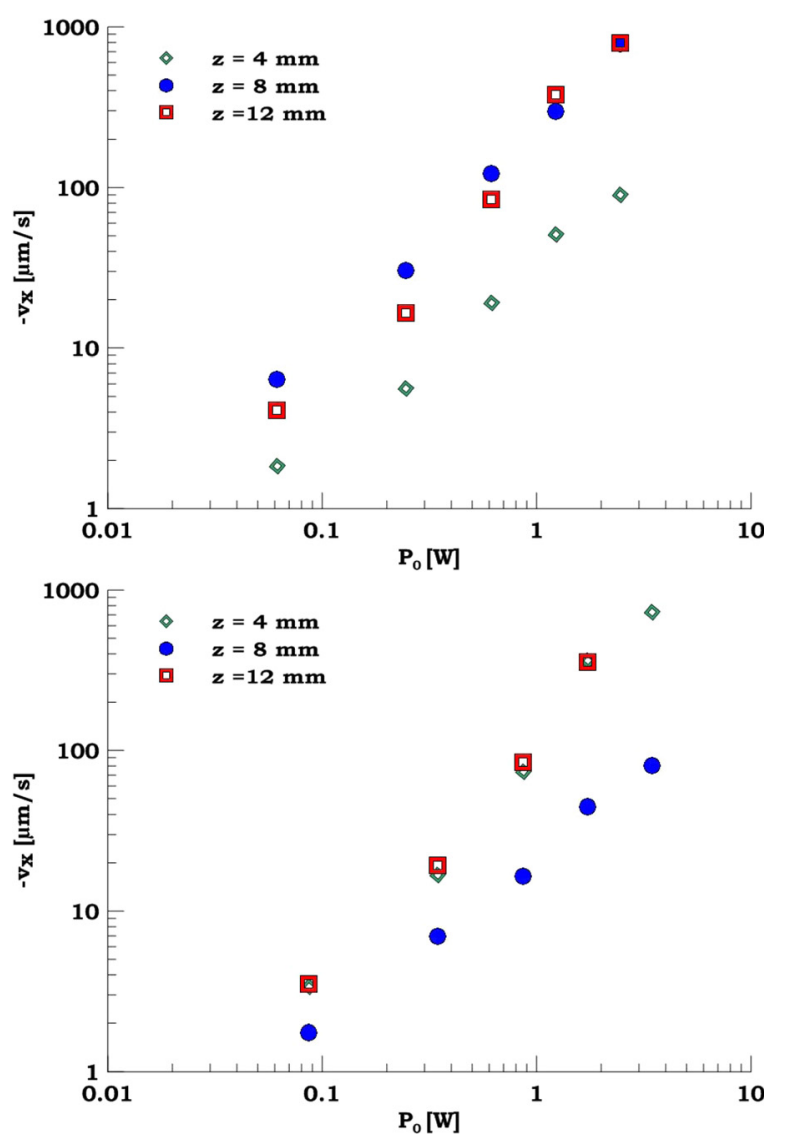

FIG. 7. (Color online) Experimental results for fluid velocity as a function of input power $P_{0}$ at height $y=80 \mu \mathrm{m}$ for different values of $z$. Upper plot: open-end configuration. Lower plot: short-end configuration.

for frequencies around $1 \mathrm{GHz}$ and conductivities smaller than $2 \mathrm{~S} / \mathrm{m}$. Therefore, the electrical force expression can be simplified to

$$
\left\langle\mathbf{f}_{\mathbf{E}}\right\rangle=-\frac{1}{4} \varepsilon^{\prime} \alpha\left|\mathbf{E}^{2}\right| \nabla T
$$

\section{B. Buoyancy force}

The gravitational body force $f_{g}$ generated by the temperature field is

$$
\mathbf{f}_{\mathbf{g}}=\Delta \rho_{m} \mathbf{g} \approx\left(\frac{\partial \rho_{m}}{\partial T}\right) \Delta T \mathbf{g}
$$

where $\mathbf{g}$ is the acceleration due to gravity. We can estimate the relative importance between gravitational and electrical forces. The ratio is of the order of

$$
\frac{f_{g}}{f_{e}}=\frac{\left(\partial \rho_{m} / \partial T\right) g \Delta T}{\frac{1}{2} \varepsilon \alpha E_{0}^{2}|\nabla T|} \sim \frac{2\left(\partial \rho_{m} / \partial T\right) g}{\varepsilon \alpha} \frac{\ell^{3}}{V_{0}^{2}},
$$

where $\ell$ is a typical distance of the system. The gradient in temperature has been approximated by $|\nabla T| \sim \Delta T / \ell$ and the electric field by $E_{0} \sim V_{0} / \ell$, with $V_{0}$ the potential amplitude of the TEM mode. Taking typical values for water, the ratio between gravitational and dielectric forces becomes $2 \cdot 10^{12} \ell^{3} / V_{0}^{2}$ in S.I. units. For a typical length $\ell=175 \mu \mathrm{m}$ (the inter-electrode gap) $f_{g} / f_{E} \sim 10 / V_{0}^{2}$, so electrical forces dominate over buoyancy for potential amplitudes greater than $3.3 \mathrm{~V}$. In our experiments both force terms should be taken into account.

\section{Electric field equations}

In the frequency range of our study, the wavelength of the fundamental mode propagating in the coplanar waveguide is larger than the transverse directions of the coplanar waveguide. Under this condition, this mode is a quasi-TEM mode, that is, the fields along the propagation direction $z$ (the longitudinal fields) can be neglected, and the transverse fields can be assumed to be quasi-static. ${ }^{15}$ Thus, the electric field can be considered as irrotational in the $x-y$ plane and, therefore, it is possible to define an electric potential that, for the advancing wave, is of the form $\phi_{+}(x, y, z)=\phi_{+}(x, y) \exp \left[\mathrm{i}\left(\omega t-k_{z} z\right)\right]$, and the traveling electric field can be written as $\mathbf{E}_{+}=-\nabla_{t} \phi_{+} \exp \left[\mathrm{i}\left(\omega t-k_{z} z\right)\right], \quad$ where $\quad \nabla_{t} \equiv \mathbf{e}_{x} \partial_{x}+\mathbf{e}_{y} \partial_{y}$ stands for transversal gradient. The wavenumber is given by

$$
k_{z}=\omega \sqrt{L C}
$$

with $L$ and $C$ the induction and capacitance per unit length of the transmission line. In this work, the wavenumber $k_{z}$ is obtained by means of an algorithm developed in the research group of some of the authors for the computation of the propagation constant in coplanar waveguides. ${ }^{16}$ The algorithm is based on a quasi-static analysis. In the range of validity of the quasi-static assumption, if the medium is nonmagnetic, the inductance $L$ is related to $C_{0}$, the capacitance without dielectrics, and $c$, the velocity of light, via the following equation: ${ }^{16}$

$$
L=\frac{1}{c^{2}} C_{0}^{-1} .
$$

Both $C$ and $C_{0}$ are obtained by imposing voltages in the conductor and computing the charge by means of the Method of Moments. $^{17}$

The transversal divergence of the displacement current is equal to zero and provides the equation for the electric potential:

$$
\nabla_{t} \cdot\left[(\sigma+i \omega \tilde{\varepsilon}) \nabla_{t} \phi\right]=0 \quad \text { with } \quad \tilde{\varepsilon}=\varepsilon^{\prime}-i \varepsilon^{\prime \prime} .
$$

Both the real part $\varepsilon^{\prime}$ and the imaginary part $\varepsilon^{\prime \prime}$ depend on frequency. The potential difference between conductors is the required boundary condition. For standing waves, this potential difference along the transmission line is of the form

$$
\begin{aligned}
& V(z)=V_{0} \sin \left(k_{z} z\right) \cos (\omega t) \text {, short end, } \\
& V(z)=V_{0} \cos \left(k_{z} z\right) \cos (\omega t) \text {, open end, }
\end{aligned}
$$

where $z=0$ is the end of the transmission line. In our system, the end is inside the microchannel.

\section{Temperature field}

The temperature field is a solution of the energy balance equation. ${ }^{8}$ For high enough frequencies, the equation of energy can be simplified to the steady-state: ${ }^{11}$ 


$$
\rho_{m} c_{p}(\mathbf{v} \cdot \nabla T)=k \nabla^{2} T+P_{\mathrm{diel}}+P_{\mathrm{ohm}}
$$

where $P_{\text {diel }}=\frac{1}{2} \varepsilon^{\prime}|\mathbf{E}|^{2}$ and $P_{\text {ohm }}=\frac{1}{2} \sigma|\mathbf{E}|^{2}$ are the timeaveraged dissipated power densities due to dielectric and ohmic losses, respectively.

The ratio of heat convection to diffusion can be written as $\rho_{m} c_{p} v \ell / \mathrm{k}$, where $v$ is the fluid velocity and $\ell$ a typical distance in the system. For a typical distance $\ell=300 \mu \mathrm{m}$, this ratio is much less than unity for velocities much less than $500 \mu \mathrm{m} / \mathrm{s}$. In our observations, velocities are much smaller that $500 \mu \mathrm{m} / \mathrm{s}$ and, therefore, heat convection can be neglected in front of heat diffusion, which reduces the equation of energy to

$$
k \nabla^{2} T+\frac{1}{2}\left(\sigma+\omega \varepsilon^{\prime \prime}\right)|\mathbf{E}|^{2}=0 .
$$

For the corresponding boundary condition, we assume that outside the device the system is at fixed room temperature.

From Eq. (10), we can see that the increment of temperature is proportional to the dissipated power, which is assumed to be equal to input power $P_{0}$. Therefore, buoyancy is proportional to input power $P_{0}$ and the flow due to dielectric forces is proportional to $E^{2} P_{0} \propto P_{0}^{2}$.

\section{E. Fluid dynamics equations}

The fluid velocity holds Navier-Stokes equations for incompressible fluids

$$
\rho_{m}(v \cdot \nabla) \mathbf{v}=\eta \nabla^{2} \mathbf{v}-\nabla p+\mathbf{f}_{\mathbf{g}}+\left\langle\mathbf{f}_{\mathbf{E}}\right\rangle, \nabla \cdot \mathbf{v}=0 .
$$

The nonlinear term $(\mathbf{v} \cdot \nabla) \mathbf{v}$ can be neglected because typically in microsystems the Reynolds number is much less than one. The boundary conditions are those of zero velocity for all the boundaries of the micro-chamber. AC induced charge electro-osmotic flow is not considered on electrodes because at signal frequencies around gigahertz the charge induced at double layers is negligible $(1 / \omega$ much smaller than the $R C$ time for charging the double layer ${ }^{18}$ ). In addition, at the dielectric walls there could be some oscillatory electroosmotic flow at gigaherz frequency. However, this frequency is so high that the induced AC flow would have negligible amplitude and extension (the diffusion layer for this oscillating flow has a thickness of the order of $\left.\sqrt{\eta / \rho_{m} \omega} \sim 0.01 \mu \mathrm{m}\right)$.

\section{NUMERICAL IMPLEMENTATION}

We solved the electric and temperature fields in a domain formed by: the glass layer, water, copper (only temperature), substrate, and air surrounding the system. Two layers of air are included in the computation domain, above the glass layer and under the substrate, to account for temperature exchange of the system with the nearby surrounding air (see Fig. 3). The velocity field is computed in the water domain.

For the numerical computations, we used the commercially available finite-elements package Comsol Multiphysics 3.5. ${ }^{19}$ We used a free mesh generation (unstructured) with extra fine mesh parameters. Model validation was done by testing the flow velocity solution when the number of mesh elements varies from 72442 (normal mesh) to 430040 (extra fine mesh).

\section{A. Boundary conditions}

To reduce computations, we first take advantage of the symmetry of the problem at plane $(x=0)$ at the center of the source electrode. Secondly, we assume periodicity in the axial direction, which is established by the electromagnetic standing waves. Since the temperature source and the electrical body force are both proportional to $E^{2}=E_{0}^{2} \cos ^{2}\left(k_{z} z\right)$, temperature and velocity fields are repeated every $\Delta z=\lambda / 2$, where $\lambda=2 \pi / k_{z}$. In addition, we take advantage that $E^{2}$ as a function of $z$ is either a maximum or minimum at $z_{n}=(\lambda / 4) n$ (with $n$ an integer), so that $z=z_{n}$ are planes of symmetry. Therefore, the domain extends in the $z$-direction from $z=0$ to $z=\lambda / 4$. The solution at any coordinate $z \geq \lambda / 4$ can be derived, by an adequate translation.

\section{B.C. for temperature}

External boundaries are set to ambient temperature $T_{0}$. Interfaces between materials are ruled by continuity condition. At the planes $(x=0),(z=0)$, and $(z=\lambda / 4)$ the symmetry condition imposes no heat flux, i.e., $\partial T / \partial n=0$.

\section{B.C. for velocity of the fluid $v(x, y, z)$}

Zero velocity is set at the walls of the microchannel. The symmetry condition at the boundary planes $(x=0)$, $(z=0)$, and $(z=\lambda / 4)$ imposes no flux of mass and momentum, i.e., $v_{n}=0, \partial v_{\|} / \partial n=0$.

\section{B.C. for potential}

The potential is solved only in the $x-y$ plane with boundary condition of $\phi=V_{0} \sin \left(k_{z} z\right)$ at the inner electrode and $\phi=0$ at the outer electrode. The outer boundaries and the symmetry plane at $x=0$ are set to condition of no flux, i.e., $\partial \phi / \partial n=0$. The value of the voltage amplitude $V_{0}$ is derived from the total power delivered to the microchannel from the microwave source. We assume that all the power delivered by the source is consumed in water (we checked experimentally that the radiated power is negligible)

$$
P=\frac{1}{2} \int_{\tau}\left(\sigma+\omega \varepsilon^{\prime \prime}\right)|\mathbf{E}|^{2} d \tau,
$$

where the integral extends overall the microchannel.

\section{B. Results}

The computed solution for temperature distribution is depicted in Fig. 8, the plot shows the increment $\Delta T=T-T_{0}$ with respect to ambient temperature $T_{0}$. The plot is for a voltage amplitude $V_{0}=5.17 \mathrm{~V}$, which corresponds to an input power equal to $385.5 \mathrm{~mW}$. The maximum expected temperature rise is $13^{\circ} \mathrm{C}$. In Fig. 9, we show a $2 \mathrm{D}$ profile of the velocity field in the plane $(x, y)$ near the source electrode 


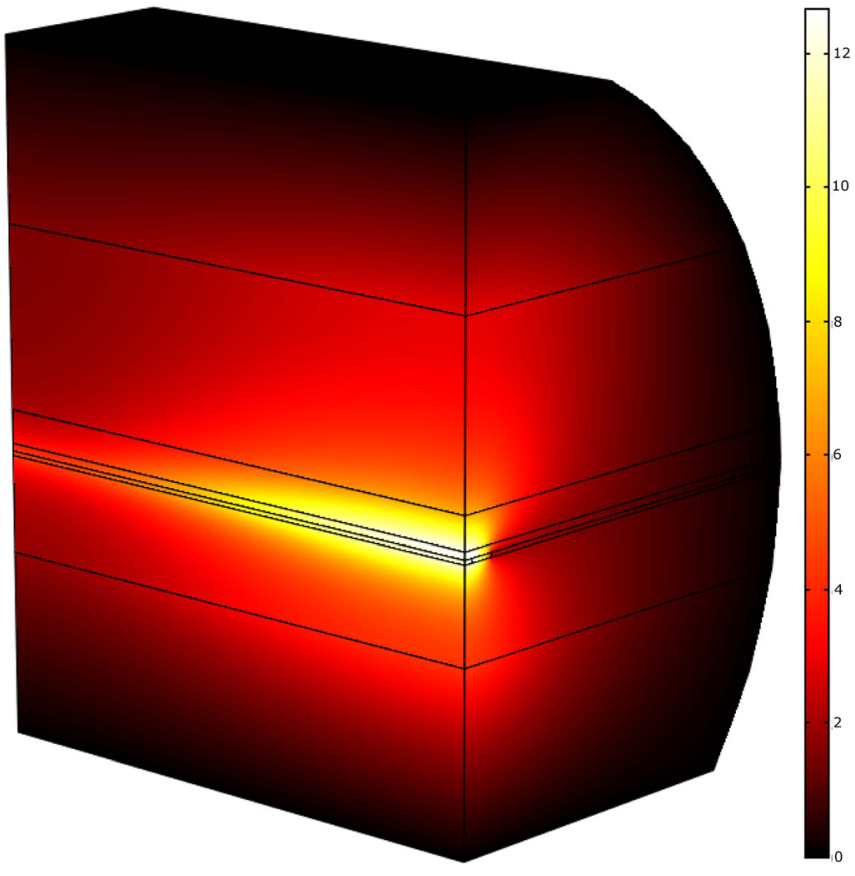

FIG. 8. (Color online) Temperature distribution. The plot shows the increment $\Delta T=T-T_{0}$ from ambient temperature $T_{0}$. Frequency: $f=3.055 \mathrm{GHz}$ and voltage amplitude $V_{0}=5.17 \mathrm{~V}$, which corresponds to an input power: $P_{0}=358.5 \mathrm{~mW}$

where flow is maximal. Similar velocity profiles are obtained for other values of frequency and voltage.

Figure 10 shows the dissipated power as a function of the voltage squared $V_{0}^{2}$ of the standing wave at different values of frequency (continuous lines). The dotted lines plot corrections to the model that take into account the variations of complex permittivity with temperature. These curves allow us to obtain the corresponding voltage amplitude to each input power under the assumption that all input power is dissipated in the microchannel by dielectric losses. A comparison of experimental and numerical results of $v_{x}$ is shown in Fig. 11 for different values of coordinate $z$, frequency, and input power. Although numerical results are close to experimental ones and have the same profile, in general, they are smaller.

\section{ANALYSIS OF APPROXIMATIONS}

In this section, we analyze the importance of the following assumptions: (a) the power consumed at the electrodes is

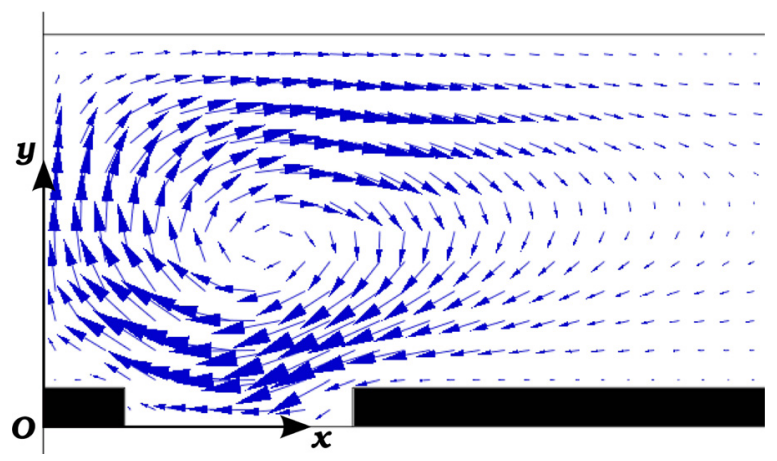

FIG. 9. (Color online) Velocity field in the plane $(x, y)$ near the source electrode, where it reaches its maximum. Frequency: $f=3.055 \mathrm{GHz}$ and and voltage amplitude $V_{0}=5.17 \mathrm{~V}$, (corresponding input power: $P_{0}=358.5 \mathrm{~mW}$ ).

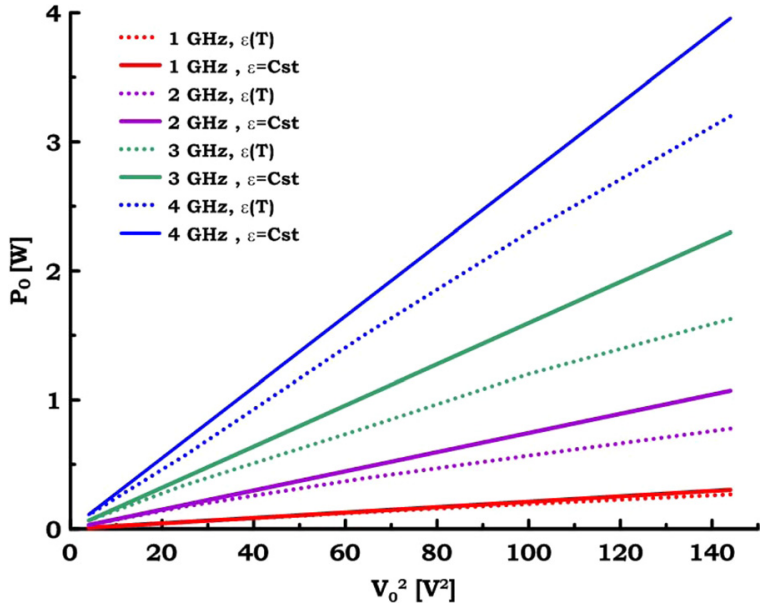

FIG. 10. (Color online) Dissipated power in the microchannel due to dielectric losses as a function of voltage squared for different frequencies. Continuous lines correspond to model with constant permittivity, while the dotted lines correspond to the corrected model with $T$-dependent permittivity.

negligible compared to the power consumed in the water bulk by dielectric losses; (b) the complex permittivity $\tilde{\varepsilon}$ was assumed to be constant with temperature in all equations except the force expression; and (c) the viscosity was assumed to be constant with temperature.

\section{A. Skin depth}

At the interface between a perfect conductor and a dielectric, the tangential component of the electric field vanishes; only the normal component exists. However, for a real conductor, a weak tangential electric field exists to support the surface currents. This surface electric field will decay exponentially inside the bulk of the conductor with a penetration length named skin depth,

$$
\delta=\sqrt{\frac{1}{\mu_{0} \omega \sigma}} .
$$

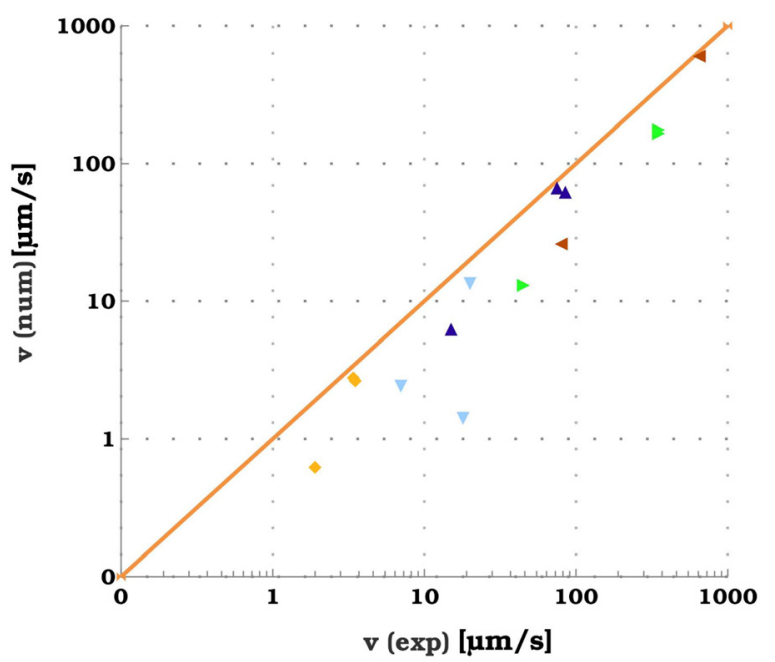

FIG. 11. (Color online) Comparison between numerical and experimental results. The plot shows velocity $v_{x}$ at different values of coordinate $z$, frequency, and input power. 
This means that a part of the microwave power is dissipated in the conductor. To check how the assumption of a perfect conductor or real conductor affects our model solution, we compute this power and compare it to the whole power consumed by dielectric losses of water. This power per unit of axial length is given by ${ }^{20}$

$$
P_{\text {cond }}=\frac{1}{2 \sigma \delta} \int H^{2} d l
$$

where the integral extends to the contour of conductors. Given the relationship between voltage and current for a transmission line, $V_{+}=Z_{0} I_{+}$, and that $I=\oint \mathbf{H} \mathbf{d l}$ around the source conductor, our computations show that the dissipated power due to the current in the conductors is less than $4 \%$ of that dissipated in the dielectric (water). Furthermore, as we will see in the next section, changes in liquid permittivity and viscosity with temperature affect the solution in a more significant manner. So we conclude that the assumption of a perfect conductor is valid.

\section{B. Variation of water permittivity with temperature}

We first investigate how the variation of permittivity with temperature affects the solution. Complex permittivity $\tilde{\varepsilon}$ written as a function of frequency and temperature, has the following form: ${ }^{21-23}$

$$
\begin{gathered}
\tilde{\varepsilon}(\omega, T)=\varepsilon^{\prime}(\omega, T)-i \varepsilon^{\prime \prime}(\omega, T), \\
\varepsilon^{\prime}(\omega, T)=\varepsilon_{\infty}(T)+\frac{\varepsilon_{S}(T)-\varepsilon_{\infty}(T)}{1+\omega^{2} \tau^{2}(T)}, \\
\varepsilon^{\prime \prime}(\omega, T)=\frac{\left(\varepsilon_{S}(T)-\varepsilon_{\infty}(T)\right) \omega \tau(T)}{1+\omega^{2} \tau^{2}(T)} .
\end{gathered}
$$

Figure 12 plots the relative complex permittivity versus temperature for different frequencies. Both the real and imaginary parts of the relative permittivity are decreasing functions of temperature.

We perform the analysis by doing an iterative calculation. First, we compute the electric field corresponding to a given input power, $P_{0}$, for homogeneous temperature. Then, the temperature field generated by dielectric heating is computed using previous solution for the electric field. We recalculate the electric field using the new values of complex permittivity and temperature field under the condition that the computed power is equal to the input power $P_{0}$. The process is repeated until the solution reaches convergence. The effect of temperature is as follows. Both $\varepsilon^{\prime}$ and $\varepsilon^{\prime \prime}$ decrease with temperature. Since $\varepsilon^{\prime}$ decreases the dielectric force decreases. On the other hand, since the power is fixed, a decrement of $\varepsilon^{\prime \prime}$ implies an increase of $E^{2}$, which leads to an increase of the dielectric force. The total change gives a decreasing dielectric force, but less than expected a priori. The decrease in the computed velocity is around $12 \%$ in the case: $f=3.055 \mathrm{GHz}$ and $P_{0}=358.5 \mathrm{~mW}$.

A change in the complex permittivity would also lead to a change in the wavenumber. However, the value comes from the squared-root of the capacitance $C$ and we can expect that the change is not very strong, as suggested by the
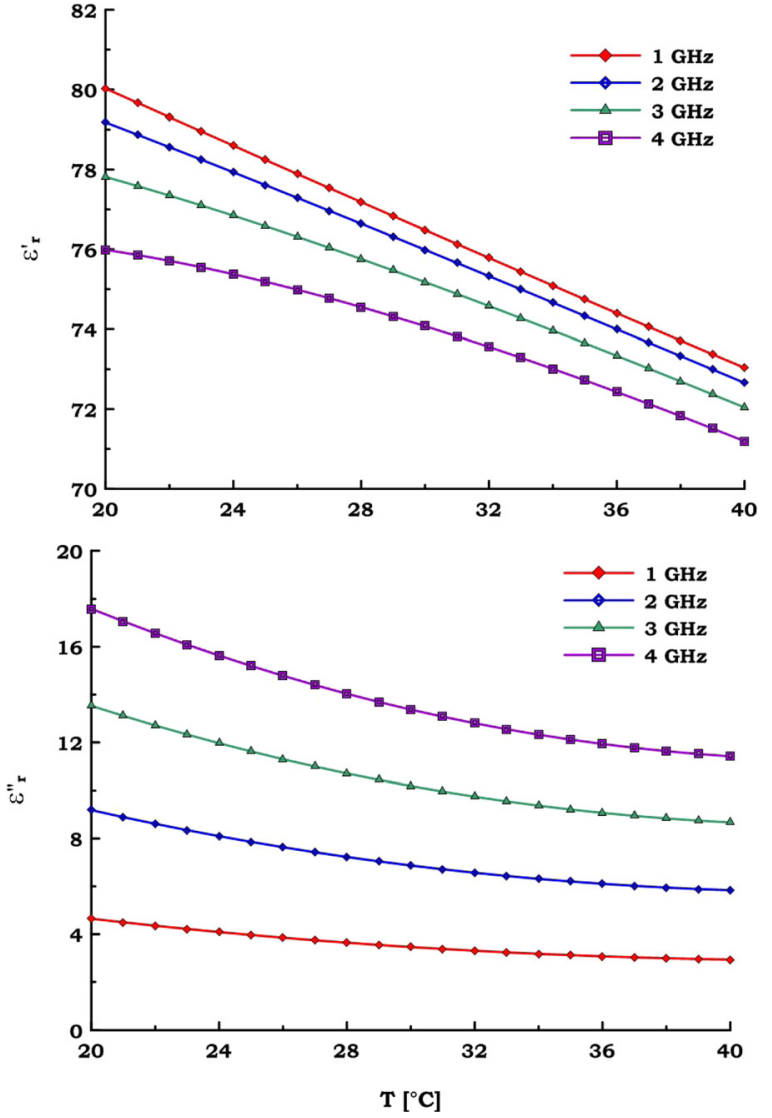

FIG. 12. (Color online) Real and imaginary parts of relative permittivity of water $\varepsilon_{r}(T)$ undergo a substantial variation from their values at ambient temperature. Here we show the variation in the range of temperature $20^{\circ} \mathrm{C}$ $<T<40^{\circ} \mathrm{C}$ and frequencies of $1,2,3,4 \mathrm{GHz}$.

small difference between the expected and the measured wavelength.

\section{Variation of viscosity with temperature}

Viscosity is the property which exhibits the most relevant variation with temperature. The variation of water viscosity with temperature has the following form: ${ }^{24,25}$

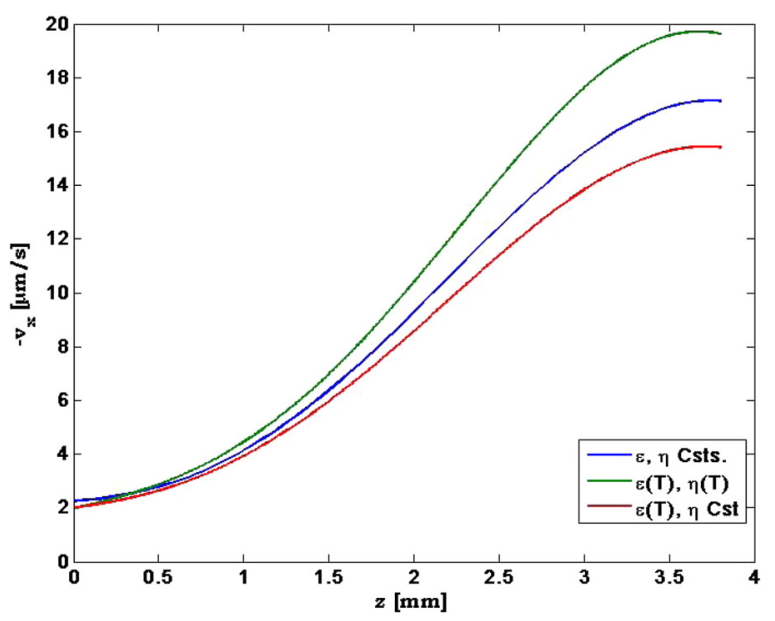

FIG. 13. (Color online) Comparison of velocity profile when permittivity and viscosity of the liquid are assumed to be constants or $T$-dependent. The plot shows velocity along the channel length at a path at the electrodes level, $f=3.055 \mathrm{GHz}$ and $P_{0}=358.5 \mathrm{~mW}$. 


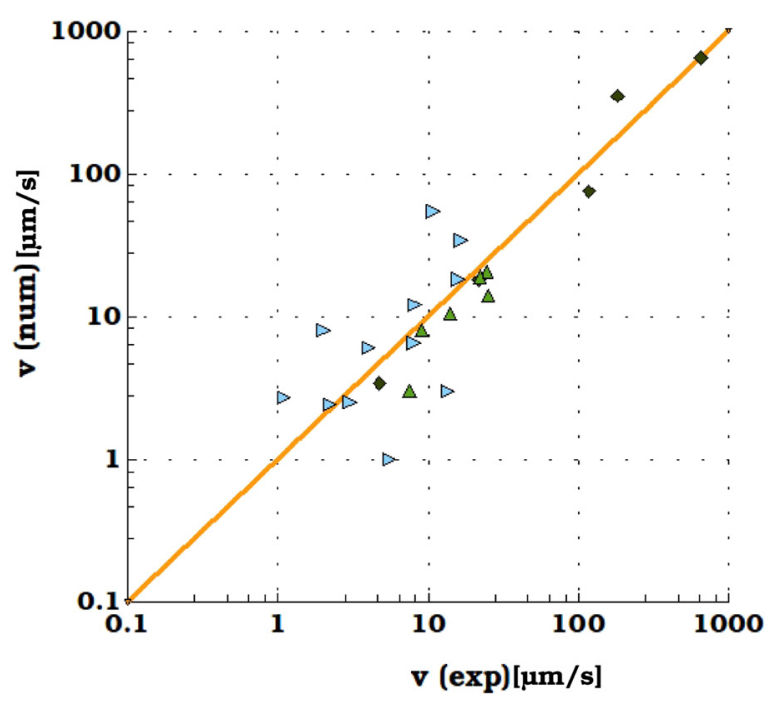

FIG. 14. (Color online) Comparison of experimental and numerical velocity values computed with the reviewed model, at different values of coordinate $z$, frequency $f$, and input power $P_{0}$.

$$
\eta(T)=A \times 10^{B /(T-C)},
$$

where $A=2.414 \times 10^{-5} \mathrm{~Pa} \cdot \mathrm{s}, B=247.8 \mathrm{~K}$, and $C=140 \mathrm{~K}$. In the range of interest (between $20^{\circ} \mathrm{C}$ and $40^{\circ} \mathrm{C}$ ) the viscosity decreases from 1 to $0.65 \mathrm{mPa} \cdot \mathrm{s}$. Including this dependence in our model, we get the solution shown in Fig. 13 for the flow velocity, where variations with $T$ of $\varepsilon^{\prime}, \varepsilon^{\prime \prime}$, and $\eta$ are all taken into account. Since water viscosity is a decreasing function of temperature, the computed velocity increases due to the rise of temperature. Results for $f=3.055 \mathrm{GHz}$ and $P_{0}=358.5 \mathrm{~mW}$, give an increase in velocity around $13 \%$ with respect to solution with $\varepsilon$ and $\eta T$-independents. At higher input power, increase in computed velocities would be even higher than measured ones, see Fig. 14. We can see that the most significant effect on the velocity amplitude is due to the viscosity dependence on temperature. Figure 14 shows that the experimental and numerical results are now coincident within experimental errors.

\section{CONCLUSIONS}

We have performed an experimental and numerical investigation of microwave-induced water flow in a microchannel built on top of a coplanar wave guide. The flow originated from standing waves in the TEM mode was studied in the frequency range $1-4 \mathrm{GHz}$. Maxima and minima of electric field amplitude (i.e., antinodes and nodes of the standing wave) led to maxima and minima of fluid flow. The measured spatial periodicity of the fluid flow pattern was in good agreement with the theoretical wavelength of the TEM mode inside the microchannel. 3D finite-element calculations of the electric, temperature, and fluid velocity fields in the microchannel were presented. The calculations were performed in the limit of small temperature variations, so that material properties are constant in all the equations, but appear in the body force (Boussinesq-like approximation). This limit also allowed us to decouple the electrical, mechanical, and thermal equations. Computed velocity field exhib- ited the same patterns found in the experiments and were in quantitative agreement with velocity data. Subsequently, the effect of considering finite increments of temperature was taken into account and the new numerical results improved the quantitative comparison with experimental velocities. In particular, the most important effect happened to be the decrease of viscosity with temperature, which led to a global increase in velocity maxima in better agreement with the experimental data.

The study of water flow originated by microwaves in microsystems is relevant to the applications of microwaves in the lab-on-a-chip technology.

\section{ACKNOWLEDGMENTS}

We acknowledge financial support from Spanish Government Ministry MEC (Contract No. FIS2006-03645) and Junta de Andalucía (Contract No. P09-FQM-4584). E. Verlage acknowledges financial support of MIT-Spain Program Internship.

${ }^{1}$ M. Mehdizadeh, Microwave/RF Applicators and Probes for Material Heating, Sensing, and Plasma Generation (Elsevier, Amsterdam, 2010).

${ }^{2}$ H. Lee and J. Yook, Appl. Phys. Lett. 92, 254103 (2008).

${ }^{3}$ G. A. Ferrier, S. F. Romanuik, D. J. Thomson, G. E. Bridges, and M. R. Freeman, Lab Chip 9, 3406 (2009).

${ }^{4}$ J. J. Shah, S. G. Sundaresan, J. Geist, D. R. Reyes, J. C. Booth, M. V. Rao, and M. Gaitan, J. Micromech. Microeng. 17, 2224 (2007).

${ }^{5}$ D. Issadore, K. J. Humphry, K. A. Brown, L. Sandberg, D. A. Weitz, and R. M. Westervelt, Lab Chip 9, 1701 (2009).

${ }^{6}$ A. Ramos, A. Robles, P. García-Sánchez, and M. J. Freire, Appl. Phys. Lett. 94, 024104 (2009).

${ }^{7}$ A. Ramos, P. García-Sánchez, A. Robles, and M. J. Freire, J. Electro. 67, 377 (2009).

${ }^{8}$ Electrohydrodynamics, edited by A. Castellanos (Springer, New York, 1998).

${ }^{9}$ ImageJ is a public domain, Java-based image processing program, located at: http://rsbweb.nih.gov/ij/.

${ }^{10}$ J. A. Stratton, Electromagnetic Theory (McGraw-Hill, New York, 1941).

${ }^{11}$ A. Ramos, H. Morgan, N. G. Green, and A. Castellanos, J. Phys. D: Appl. Phys. 31, 2338 (1998).

${ }^{12}$ N. G. Green, A. Ramos, A. González, A. Castellanos, and H. Morgan, J. Phys. D: Appl. Phys. 33, L13 (2000).

${ }^{13}$ A. González, A. Ramos, H. Morgan, N. G. Green, and A. Castellanos, J. Fluid Mech. 564, 415 (2006).

${ }^{14}$ CRC Handbook of Chemistry and Physic, Internet version, edited by David R. Lide, 2005.

${ }^{15}$ D. M. Pozar, Microwave Engineering, 3rd ed. (John Wiley \& Sons, Hoboken, NJ, 2005).

${ }^{16}$ F. Medina, E. Drake, and M. Horno, IEEE Trans. Microwave Theory Techniques 42, 2328 (1994).

${ }^{17}$ R. F. Harrington, Field Computation by Moment Method (IEEE, Piscataway, 1993)

${ }^{18}$ A. Ramos, H. Morgan, N. G. Green, and A. Castellanos, J. Colloid Interface Sci. 217, 420 (1999).

${ }^{19}$ Comsol Multiphysics is a software package for finite element analysis with website: http://www.comsol.com.

${ }^{20} \mathrm{~S}$. Ramo, J. R. Whinnery, and T. Van Duzer, Fields and Waves in Communication Electronics (Wiley, New York, 1965).

${ }^{21}$ R. Somaraju and T. Trumpf, IEEE Trans. Antenna and Propagation 54(11), 3441 (2006)

${ }^{22}$ A. Catenaccio, Y. Daruich, and C. Magallanes, Chem. Phys. Lett. 367, 669 (2003).

${ }^{23}$ R. Buchner, J. Barthel, and J. Stauber, Chem. Phys. Lett. 306, 57 (1999).

${ }^{24}$ R. C. Reid, J. M. Prausnitz, and B. E. Poling, The Properties of Gases and Liquids, 4th ed. (McGraw-Hill, New York, 1987).

${ }^{25}$ The International Association for the Properties of Water and Steam: http://www.iapws.org. 